\title{
Erratum
}

\section{Successsive resonance-enhanced two-photon ionization of elements abundant in nebulae}

\author{
I. Atoms and ions of $\mathrm{C}, \mathrm{N}$, and $\mathrm{O}$ \\ S. Johansson ${ }^{1}$ and V. S. Letokhov ${ }^{2,1}$ \\ 1 Lund Observatory, Lund University, PO Box 43, 22100 Lund, Sweden \\ e-mail: Sveneric.Johansson@astrok.lu.se,vladilen.Letokhov@astro.lu.se \\ 2 Institute of Spectroscopy, Russian Academy of Sciences, 142190 Troitsk, Moscow region, Russia
}

A\&A, 375, 319-327 (2001), DOI: 10.1051/0004-6361:20010738

In the title of this paper we used the term "nebulae", with which readers associate certain numbers concerning temperature and density. The radiative process we discuss is more applicable to denser gas condensations (blobs) located closer to hot, luminous stars, e.g. the enigmatic Weigelt blobs in the vicinity of $\eta$ Car. A more relevant title should be: "Successive resonance-enhanced two-photon ionization of elements abundant in gas condensations close to hot stars. I. Atoms and ions of C, $\mathrm{N}$ and $\mathrm{O}$ ”.

Acknowledgements. We acknowledge Prof. G. Ferland for useful comments. 